\title{
La Inestable Aceptación de la Homosexualidad. El Caso de las Escuelas Católicas de Elite en Santiago de Chile
}

\author{
The Unstable Acceptance of Homosexuality. The Case of Elitist \\ Catholic Schools in Santiago de Chile
}

\author{
Pablo Astudillo Lizama * \\ Université Paris V Descartes
}

\begin{abstract}
Dentro de las escuelas católicas de élite, el tratamiento de la homosexualidad es el resultado de una conciliación entre el mandato contemporáneo de asegurar una sexualidad coherente en los individuos y las normas eclesiales en las que se enmarcan sus proyectos educativos. En apariencia, estas escuelas serían hostiles al reconocimiento de las sexualidades "fuera de la norma", no obstante, distintos factores permiten la emergencia de un tratamiento "comprensivo" de la sexualidad homosexual. Dicha "comprensión”, sin embargo, opera en realidad sobre una reflexividad limitada respecto al proceso de individuación sexual lo que redunda en un reconocimiento parcial del individuo homosexual. Aquí se esconden todavía algunas trampas que vale la pena atender. De alguna forma, lo que observamos en este espacio es también parte de un proceso social general donde el avance de determinadas formas políticas de aceptación de la homosexualidad convive a veces con una estructura de la homofobia más sutil, cuya real erradicación requiere ir más allá de la comprensión aparente del "otro" que hoy domina este espacio social.
\end{abstract}

Descriptores: Educación sexual, Escuela católica, Homosexualidad, Individuación, Reconocimiento.

Within elitist catholic schools, homosexuality is treated as the result of conciliation between contemporary norms guaranteeing an individual's coherent sexuality, and ecclesiastic rules defining a school's educational program. Supposedly, catholic schools would be hostile towards non-dominant sexualities; however, different factors contribute to the emergence of an "accepting" treatment of homosexuality. That "acceptance", nevertheless, is based on limited reflexivity regarding the process of sexual individuation. As a result, there is partial recognition of the homosexual person, and it is important to expose the hidden social traps that prevent a complete recognition from taking place. In a way, what we observe in catholic schools is also part of a much larger social process. The advance of political forms of acceptance towards homosexuality sometimes coexists with a much subtler structure of homophobia, whose eradication requires going beyond the apparent acceptance of the "other" that presently dominates this social space.

Keywords: Sexual education, Catholic school, Homosexuality, Individuation, Recognition.

Este artículo se enmarca dentro del Proyecto Anillos en Ciencias Sociales y Humanidades, "Normalidad, Diferencia y Educación" (SOC1103), el cual contó con el financiamiento del Programa de Investigación Asociativa-Comisión Nacional para la investigación Científica y Tecnológica (PIA-CONICYT).

\footnotetext{
*Contacto: pablo.astudillo.lizama@gmail.com Recibido: 05/08/2016

ISSN: 0718-7378

$1^{\text {a }}$ Evaluación: 15/08/2016

www.rinace.net/rlei/

Aceptado: 24/09/2016
} 


\section{Revisión de la literatura}

El tema de la visibilidad o invisibilidad de la diversidad sexual en la escuela ha sido ampliamente abordado por numerosas investigaciones, orientadas la mayor parte de las veces a la descripción de los mecanismos de reproducción de la homo, lesbo y transfobia en el espacio escolar y las consecuencias de dicha hostilidad sobre los individuos del colectivo LGBT (Cáceres y Salazar, 2013; Generelo y Pichardo, 2006) Asimismo no son pocos los estudios que han abordado la cuestión de cómo la homosexualidad se constituye como un elemento que -por oposición, por negación- construye la heterosexualidad, especialmente la masculina (Ceballos, 2012; Devis, Fuentes y Sparkes, 2005; Martino, 1999; Renold, 2004). Por esta misma razón queremos situarnos frente a esta problemática de un modo diferente.

Con este propósito observaremos las lógicas de posicionamiento del individuo homosexual dentro del espacio escolar, toda vez que, contrariamente a la representación de las instituciones católicas como hostiles a la homosexualidad, encontramos en ella un discurso de aceptación de dicha orientación sexual. No obstante aquello, es interesante constatar que el tratamiento cuidadoso de la diferencia esconde también la confirmación de una norma dominante, fundamental para comprender cualquier proceso de individuación sexual. Dicha norma que tiene que ver con el trabajo que hace el individuo sobre sí mismo, sigue situando a la homosexualidad en un plano de subordinación que supuestamente se trata de combatir.

El presente artículo se enmarca dentro de un trabajo de investigación doctoral titulado "Tensiones y paradojas de un proceso de individuación sexual. El caso de las escuelas católicas de élite en Santiago de Chile". Surge a partir de la constatación que la salida del clóset de algún estudiante y el consecuente tratamiento cuidadoso de su homosexualidad constituyen casi siempre la primera mención frente a la pregunta sobre como las escuelas atienden las situaciones "fuera de la norma" (aquellas cuestiones "inesperadas" por sus planes de educación sexual). Dicha respuesta, además, es frecuentemente movilizada como la prueba del progresismo de un establecimiento, de su adecuación al mundo contemporáneo y de su mayor apertura frente a los cambios de la sociedad chilena.

Interpretamos entonces este fenómeno dentro de un marco más general de comprensión de la sexualidad humana y de la gestión de las normas que la regulan hoy en día. Veremos que al examinar esta operación con más detalle podremos comprender como dicha aceptación o apertura frente a la homosexualidad depende de un trabajo de posicionamiento del otro, donde la comprensión total del individuo homosexual nunca es completa. Esta aceptación difusa de la homosexualidad no a nuestro juicio resultado de un "doble estándar" de la escuela sino más bien de este ejercicio de modo de situar activamente la alteridad, lo cual permite un tratamiento paternalista del individuo homosexual.

Para profundizar en esta idea revisaremos tres cuestiones fundamentales: la manera de normalizar una identidad sexual en un espacio aparentemente adverso, la forma de tratarla dentro del currículo de educación sexual en la escuela y el modo paternalista de cuidar la "diferencia" resultante. En este sentido, más que una crítica abierta, este artículo pretende ser un texto para reflexionar en torno a las operaciones que tal vez involuntariamente siguen atentando contra ese ideal inclusivo expuesto en el discurso. 


\section{Metodología}

Antes de entrar en materia presentaremos aquí los principales aspectos del trabajo de campo. El mismo se desarrolló en Santiago de Chile entre agosto de 2013 y junio de 2015 principalmente en colegios católicos que atienden a familias de altos ingresos de la ciudad. En relación a este artículo la información es obtenida por medio de una serie de técnicas cualitativas, entre las cuales se cuenta un corpus de 53 entrevistas en profundidad las cuales, de acuerdo a las características de los entrevistados, se desglosan en 39 informantes claves en instituciones educacionales (profesores, psicólogos, orientadores, directivos en 15 establecimientos educacionales) y 14 en otras organizaciones anexas (Ministerio de Educación y organizaciones privadas que imparten programas de educación sexual). En paralelo, se realizaron dos grupos focales con estudiantes de dos colegios separados, los cuales reunieron un total de 23 participantes. Toda esta información, para efectos del presente trabajo, fue analizada siguiendo la metodología de análisis del discurso.

Las reflexiones del presente artículo se construyen a partir de las respuestas a una pauta semiestructurada de entrevista, la cual abordaba diferentes temas respecto a cómo se toman decisiones para la elaboración de programas curriculares, cuáles eran las dificultades para la implementación de dichos programas en la escuela, cuál era la participación de las familias o como el establecimiento afrontaba ciertas contingencias, entre otros aspectos relativos a la educación afectiva y sexual en las escuelas. Considerando lo anterior, tomamos en cuenta los planteamientos de Canales (2013) respecto a comprender las relaciones entre la palabra personal y la palabra del grupo y los diferentes registros que, por consecuencia, pueden existir dentro de un mismo interlocutor. En el caso del tratamiento de la diversidad sexual en general, y de la homosexualidad en particular, esto es claro en la medida que se aprecian ciertas ambigüedades, por ejemplo en el modo como el sujeto describe su rol y su propia posición moral. Como consecuencia se puso especialmente atención en aquellos nudos críticos que dan cuenta del posicionamiento y tensiones de los sujetos en términos normativos, más que en la descripción detallada de sus propios comportamientos. Esto es especialmente importante al considerar mi propia situación como observador externo a la escuela y por lo tanto continuamente posicionado como tal. El límite que esta aproximación tiene -aún si se complementó con observaciones etnográficas- es la imposibilidad de acceder a la conducta final de los entrevistados y quizás a los núcleos más duros de la homofobia en la escuela.

No obstante aquello, lo que se "dice" sobre la homosexualidad da cuenta no sólo de una subjetividad individual e incluso grupal, sino que revela sobre todo las modificaciones normativas que han ocurrido a este respecto y que permiten interpretar la ambigüedad con la que la escuela trata la diferencia. Seguimos aquí los planteamientos de François Dubet (2010) y de Joan Scott (2014), respecto a la conveniencia de ir más allá de la evidencia concreta (aquello que efectivamente ocurre en interacciones a las cuales no siempre se tiene acceso directo) para abordar las representaciones de la experiencia, es decir, aquellas descripciones de la sexualidad homosexual donde el hablante identifica, critica o incluso produce estratégicamente normas sobre ella.

Es importante señalar que la selección de establecimientos se realizó a partir de diferentes contactos que fueron facilitados entre los mismos entrevistados a la manera de un muestreo "bola de nieve". A medida que dicha red se fue ampliando fue necesario 
incorporar algunas distinciones que facilitaran la comparación ad intra de la información recogida. Seguimos para efectos del análisis la diferenciación propuesta por Madrid (2013) que distingue entre colegios católicos tradicionales -aquellos fundados principalmente entre el siglo XIX y primera mitad del siglo XX por congregaciones europeas (Compañía de Jesús, Verbo Divino, Sagrados Corazones, Holly Cross, etc.) y que habrían tenido gran influencia en la formación de élites hasta 1973- y colegios de nuevos movimientos católicos, aquellos de propiedad o fuertemente influenciados por las organizaciones como el Movimiento apostólico de Schöenstatt, los Legionarios de Cristo y el Opus Dei, los cuales fundan colegios especialmente a partir de los años 1980 y que se caracterizan por su naturaleza conservadora. Ambos tipos de instituciones se diferencian de los establecimientos laicos de élite, también incluidos en este estudio a modo de un pequeño grupo de control, pero que no forman parte de las citas utilizadas en este texto.

Si bien dicha distinción nos es útil para comparar dos aproximaciones a un tema que probará ser complejo (pues de algún modo es movilizada por las propias instituciones para situarse en relación al discurso vigente del magisterio eclesial), es menester señalar que al final ambos tipos de organizaciones no logran desarrollar una aproximación a la sexualidad homosexual reconociendo en su totalidad al individuo que la encarna. Sobre tal problemática trata el siguiente texto.

\section{Discusión}

\subsection{Normalizar lo que antes era "anormal"}

En la actualidad, el posicionamiento de los individuos homosexuales dentro de la escuela católica de élite no puede ser comprendido si no se considera una serie de variables que podemos llamar estructurales, respecto de las cuales se enmarca el discurso de los actores y su capacidad reflexiva. Nos referimos con esto a las normas con las que se comprende la sexualidad de los individuos en un espacio social cualquiera, normas generales que son también recogidas por varios aspectos del currículo formal e informal presente en los establecimientos observados, independientemente del hecho que hagamos referencia a la homosexualidad o no.

No podemos entender el discurso en torno al individuo homosexual si no consideramos primero la noción de "coherencia sexual" desarrollada por Michel Bozon (2001a, 2001b, 2009). Para el autor, las normas que regulan la sexualidad son crecientemente atribuidas y sujetas a la responsabilidad individual la cual ha reemplazado la antigua tutela de la Iglesia y del Estado como agentes de control del comportamiento sexual. La internalización del control de la sexualidad por parte de los sujetos implica que aquellos son ahora los que deben dar cuenta de lo que podríamos denominar "una propia gramática sexual coherente". Con esta última idea nos referimos a la forma como se ordenan cuestiones como la elección de pareja, el modo de iniciar o terminar una relación sexual, o la manera de atender al propio deseo, entre otros elementos de la vida afectiva, todas las cuales pasan a estar sujetas a un permanente escrutinio tanto propio como ajeno (Singly, 2010).

Ahora bien, aun si esta coherencia parece ser un asunto puramente personal, en la práctica es el resultado de un proceso de socialización complejo. Para autores como Bozon (2009), Illouz (2015) o de Miguel (2015), el siglo XX inauguraría un nuevo 
escenario para la comprensión de la sexualidad al ofrecer una disociación práctica entre sexualidad y fecundidad así como un nuevo marco de comprensión del individuo. Esto termina por definir una nueva reflexividad en torno al cuerpo así como nuevos mecanismos de atención sobre él, consolidándose el placer como principio motor de la sexualidad. A lo anterior le sigue la emergencia de nuevas estrategias para administrar, mantener y renovar el deseo sexual, así como la diversificación de nuevas tecnologías que abren nuevos espacios sociales a la expresión de la sexualidad. Quizás el impacto más visible de estos nuevos principios de organización sea tanto la aparición de múltiples estándares en relación a los recorridos afectivos y sexuales (especialmente durante la vida adulta) como también la pérdida de potestad del matrimonio como única institución que asegura el acceso a la vida sexual (Kaufmann, 2011; Singly 2000).

Es este marco lo que posibilita, a partir de los años 1970, el surgimiento de "nuevas" normalidades en materia de sexualidad. Particularmente en el caso de la homosexualidad, eso repercute en una politización de los grupos LGBT así como también en la despatologización de esta forma específica de atender al propio deseo sexual. En el presente y dentro de las escuelas católicas -que es lo que nos convoca en este artículo- lo anterior implica que deje de hablarse de pecado cuando se hace referencia a la homosexualidad. Dicha retórica es reemplazada, en cambio, por un lenguaje "comprensivo" pues la sexualidad del individuo es un rasgo identitario innegable para su coherencia.

Ponte tú... un alumno que ahora está en segundo medio que en octavo empieza a hacer su proceso de salida de clóset con el psicólogo del colegio y con profesores implicados [con él]. En el fondo hay un caso que se aborda de modo súper acotado, en el fondo si sus compañeros no lo saben, él decide cuándo, yo creo que ahí depende de los alumnos cómo viven esas situaciones. Pero en general la posición del colegio es de acogida de mucho acompañamiento y de mucho respeto a la persona del alumno. No sé po, siempre somos más aguja en psicología y preguntamos 'oye ¿y qué pasa si un día llega a la fiesta de graduación un compadre con su pololo?' y claro uno empieza a incomodar po, entonces cachai que no está tan normalizado ni naturalizado. (Psicólogo, colegio católico tradicional)

De hecho ayer nos estábamos planteando que un tema urgente es la diversidad sexual. Mira me pasa que tenemos un ex alumno que abiertamente, por redes sociales, es obvio que declaró su homosexualidad y es un gallo que tu decís 'haberlo conocido toda la vida! mejores notas, alumno destacado, representante estudiantil!'(...) Todo esto generó toda una preocupación por el tema. Los alumnos, yo sentí que lo tomaron con mucha generosidad pero, sin embargo a mí me queda una inquietud, me quedé pensando y me dije: qué lata que sea porque es él porque para atrás no tenemos muchos [casos] y nos hemos hecho cargo [sólo] porque han salido del closet y no generó tanta resonancia en la comunidad, pero él sale y [produce] mucho rumor. Asi también niñitas que son lesbianas ¿̨te fijai? entonces me queda como el desafío de chuta porqué. Alguna vez salió una alumna nuestra en una revista y en la entrevista ella dice que es lesbiana, eso generó muchas cosas, y se decía: ¿por qué? si era obvio, ¿̇por qué lo cuenta? ¿por qué nombra el colegio? eso habla mal de nosotros', cosas así. Bueno es su experiencia. (Profesora colegio católico tradicional)

Sistemáticamente, el trabajo de campo arrojó que cuando se trata de abordar la homosexualidad de algún sujeto, la actitud debe ser la "acogida", la "comprensión", el "cuidado", términos que se ubican en un espacio semántico opuesto al "rechazo", el "castigo", la "corrección". Este tipo de discurso suele ser identificado como una prueba del ajuste del propio proyecto educativo con la "realidad del mundo", una forma de posicionamiento de la escuela en contraposición a un conservadurismo que insistiría en 
la sanción y la construcción de una sexualidad "ajena a lo que pasa hoy en día". Diferentes operaciones que sobrepasan el tema tratado en este artículo, probarán que la definición de "lo que es actual" es algo variable, en la medida que existe una suerte de ensimismamiento de la escuela que tiende a considerar su propia realidad como representativa de la universalidad, al menos de lo que ocurre para la clase social en la cual se ubica.

Ahora bien, las citas anteriores revelan adecuadamente algunas distinciones que aparecen cuando se trata de considerar la capacidad de agencia del individuo homosexual. Si el estudiante decidiera hacer presente su orientación en un acto tan banal pero a la vez definitorio de la identidad escolar como la graduación de fin de año, o conceder una entrevista en un periódico identificando en un mismo texto su homosexualidad y el colegio donde estudió, el tratamiento "inclusivo" comienza a mostrar algunas fisuras. Creemos que la actitud descrita en las citas puede ser entendida en virtud del desplazamiento de la homofobia desde el estigma de un sujeto hacia un terreno de las relaciones entre los individuos. En el caso de Chile y desde un punto de vista político Jaime Barrientos (2015) describe con claridad como la homosexualidad es validada crecientemente en el espacio político, lo cual se demuestra tanto en la creciente aprobación que en distintas encuestas goza la afirmación "la homosexualidad es normal y natural”, como también en el mayor grado de acuerdo que suscita la legislación en torno al matrimonio igualitario, todas tendencias que en mayor o menor grado se observan en otros países de la región. No obstante aquello, sostiene Barrientos, esta mayor aceptación convive con una suerte de rechazo micro-político que se expresa en ideas como "un verdadero hombre solo tiene relaciones con mujeres" "no debiera permitírseles a los homosexuales trabajar con menores de edad" o "a los hombres homosexuales se les debería prohibir adoptar hijos o hijas”. De algún modo las objeciones aparecen cuando se hace referencia a las relaciones que comprometen directamente al sujeto que habla.

Ahora, yo creo que igual hay profes, que uno cacha, que pueden ser homosexuales y son súper queridos y nadie se va a meter. Ahora bien, la sociedad no está bien todavía para decir, 'ah, ya bien'. No, no estamos en esa sociedad y menos acá [se refiere a su colegio], pero tampoco hay homofobia en el ambiente, para nada, te lo digo yo. A mi cuando me ha tocado hablar personalmente con alumnas que me han preguntado, qué sé yo, me impresiona positivamente la actitud de los alumnos, como súper abiertos a la diversidad. Somos un colegio que tiene integración, hace muchos años ya, nos falta para ser un colegio inclusivo, obvio. Pero es un colegio que ya hace mucho tiempo, casi en todas las salas hay niños con capacidades diferentes, eso ya te hace como un ambiente desde todo más tolerante, en este colegio hay evaluaciones diferenciadas, hay adaptaciones curriculares, entonces no sólo en el ámbito de tu orientación sexual, sino que tratamos de ser inclusivos. (Psicóloga, colegio nuevos movimientos católicos)

La postura del colegio en ningún momento ha sido como de discriminar las conductas homosexuales, no sé po, los adultos acá lo podemos tener como súper claro. Pero dentro de la cultura de los papás, de algunos papás que son más antiguos y que han tenido por años a sus hijos acá y les quedan los más chicos, [ellos] son como más conservadores. (...) Nuestro proyecto educativo es que somos inclusivos, entonces no podemos hacer discriminación: ni por apariencia, ni clase por social y confiamos de que si [los estudiantes homosexuales] llegan acá es porque necesitan ayudan, la vamos a brindar dentro de nuestras posibilidades, pero ahora nos vemos complicado. (Profesora, colegio católico tradicional)

El tema de la sexualidad ha cambiado los últimos años, yo me acuerdo hace ocho o nueve años si tú hablabas abiertamente contra la homosexualidad en una clase encontrabas aprobación total, digamos, hoy día 'ya... y por qué', 'yo no estoy tan de 
acuerdo'. Qué bueno, porque es lógico, me parece muy bien que ahora pregunten, antes así como idiotas, me gusta que tengan ese cuestionamiento. Ahora yo creo que de esa aprobación que tiene hacia la homosexualidad o el rechazo hacia alguien que opina lo contrario es influencia del ambiente... que hace que no puedas repetir el mismo mensaje que estás diciendo hace diez años. La reacción que encuentras frente a ese mensaje... como que te quedas en la forma y te quedas en ese rechazo casi visceral que ahora produce el homofóbico y no vas a la visión de fondo que es lo importante: qué es el sexo, para qué es su fin, qué es el matrimonio cuáles son sus fines. Creo que esa es la discusión importante. (Profesor, colegio nuevos movimientos católicos)

Las citas deslizan desde ya la existencia de un "límite" a dicha comprensión del otro. El tratamiento de la homosexualidad convive con otras operaciones de etiquetamiento de la diferencia que de algún modo permiten hablar de la homosexualidad más bien desde un posicionamiento moral o político, pero no testimonial. En las primeras citas la homosexualidad queda asociada a otras categorías de la inclusión, sin que en dicho proceso se cuestione la posición de la normalidad desde la cual un profesional habla, generando con ello una trasmisión irreflexiva de la noción de la diferencia (Apablaza, 2015; Matus e Infante, 2011). En la última cita, en cambio, la cuestión tiene más bien que ver con la oposición entre aceptación de la homosexualidad y la homofobia, vista positivamente por el hablante (implica un juicio reflexivo de los alumnos dice el profesor); no obstante aquello, la vigilancia sobre la homofobia dificultaría la discusión sobre lo que sería realmente importante: el matrimonio heterosexual. Es evidente como dicha operación implica una abierta marginalización de la sexualidad homosexual.

Luego, aunque no pueda hablarse abiertamente de homofobia -pues la escuela en conjunto con la política comienza a vigilar esta actitud en sus integrantes- aquella de algún modo todavía subsiste. Puede ser inconveniente utilizar aquí la definición clásica del "miedo al homosexual" (Borrillo, 2001) pues lo que se aprecia es más bien una suerte de múltiples significados que hacen cada vez más difícil de distinguir la homofobia en el discurso. A esto debemos sumar el hecho que, de acuerdo con Fassin (2008) la homofobia deja de responder a un solo patrón general sino que varía de acuerdo con las relaciones cotidianas que cada individuo establece. Dichas relaciones, sostiene el autor, están fuertemente influenciadas por la posición que el mismo tenga dentro de un determinado espacio social. Así por ejemplo, en contextos de alto capital sociocultural como el que observamos en esta investigación lo que se suele manifestar no sería la clásica violencia homofóbica atribuida a los contextos populares (aparentemente más violentos en términos físicos) sino más bien una sofisticación de los argumentos para la exclusión o inferiorización de los individuos homosexuales.

Pensemos que nuestro contexto de estudio se caracteriza por una orientación hacia la formación del carácter (Thumala, 2007) lo cual redunda también en el plano de la sexualidad, bajo la premisa de construir un individuo responsable de sí mismo, reflexivo. Es el efecto de este componente normativo lo que pasamos a examinar a continuación.

\subsection{Una cuestión de reglas}

En el plano institucional, las operaciones que hemos observado se consolidan mediante una lógica de personalización de la sexualidad. En dicha lógica convergen tanto la política pública de educación sexual, como también el discurso magisterial sobre el sexo. Entre ambos contenidos, la escuela católica define planes de formación que finalmente abordan la cuestión homosexual sin considerar la complejidad de las relaciones sociales que dibujan la individuación sexual y el propio posicionamiento de los hablantes. 
Para avanzar en nuestra reflexión es importante constatar que, de acuerdo con la ley chilena (Ley 20.418), las escuelas deben garantizar la información sobre reproducción y prevención de enfermedades de transmisión sexual, acorde a los valores con los que cada familia decida educar a sus hijos. Esto permite que una institución diseñe su propio currículo en materia de educación sexual, o bien adquiera alguno de los múltiples programas ofrecidos en el mercado, incluso en el extranjero (como constatamos en una red de colegios de nuevos movimientos católicos). De acuerdo con Palma, Reyes y Moreno (2013) esta política es consecuencia de la adaptación al principio constitucional de libertad de enseñanza que a su vez consagra el principio neoliberal de elección de los sujetos, como base de organización del currículo. Señalan los autores que es esta disposición la que permitió, por primera vez en la historia, que proyectos católicos entren en la política pública de educación sexual (como lo demuestra el hecho que tres de los siete programas propuestos por el gobierno en 2012 para ser adquiridos con financiamiento SEP tengan una abierta adscripción con el discurso magisterial). Esta diversidad de programas, no obstante, no se entendería si no existiera algún principio unificador que permitiera su transacción en el mercado: la convergencia sobre esta idea de educar a un individuo responsable de sí mismo y de sus decisiones. Como consecuencia, la educación sexual en los colegios observados se define siempre como "reflexiva".

Es este el punto que permite la viabilidad de los proyectos católicos en el plano de la educación sexual. Desde el pontificado de Juan Pablo II se ha desarrollado lo que se denomina una "ética personalista", cuestión convergente con la norma de la coherencia sexual que ya hemos examinado (Sevegrand, 2002). La diferencia aquí reside en que el magisterio apunta hacia una ética sexual se relaciona aquí con la donación interpersonal, donación que solo se entiende en el marco de cuerpos complementarios (una relación heterosexual). No hace falta profundizar en este aspecto, pero podemos señalar que de acuerdo con el discurso predominante en la escuela católica, una sexualidad ordenada, protegida y conyugal, siempre es presentada como una fuente de mayor satisfacción en relación a un comportamiento desordenado o desprotegido. Aún si el discurso es comprensivo con la homosexualidad, lo será siempre y cuando la representación de la misma apunte a la reproducción de una relación cuidadosa de pareja. No hace falta decir que difícilmente esto se puede lograr tempranamente en un espacio que favorece innegablemente el encuentro heterosexual (como se comprueba por ejemplo con la regulación de las expresiones de afecto al interior de los establecimientos).

Independiente del programa elegido o elaborado, el mismo termina siempre por fijar un marco normativo concreto para los diferentes actores en la escuela. De acuerdo con la evidencia recogida, la homosexualidad tiende a ser presentada la mayor parte de las veces como un "tema", como una "distinción" sobre la cual se puede abrir un debate que contrapone información con posiciones morales. Este último punto en particular, corrobora la tendencia descrita por Cáceres y Salazar (2013). De acuerdo con los autores, siempre se habla de la homosexualidad como una "cuestión de individuos" -es decir, de un modo encapsulado o remitido a casos que suceden- respecto de los cuales además hay un discurso ambiguo de tolerancia que se traduce en que se acepta una orientación sexual no heterosexual siempre y cuando no sea demasiado evidente o problemática.

Aun cuando exista un consenso respecto a evitar la discriminación, en general, dos son las posturas con las que se enfrenta la discusión que acabamos de mencionar. Desde un punto de vista más bien conservador la homosexualidad es presentada sobre todo como 
una categoría esencialmente distinta a la heterosexualidad que, aunque requiera el uso de cierto lenguaje inclusivo, solo puede ser visible en la medida que quede absolutamente claro que el comportamiento homosexual no es aquello que realmente se espera de un sujeto.

Las distintas neuronas que están presentes durante la adolescencia moldean el cerebro femenino y masculino de distintas maneras y con ciertas respuestas. Entonces por ejemplo, por qué las mujeres tienen mayor lenguaje que los hombres, porque el estrógeno hace que algunas áreas del cerebro no involucionen, sino que proliferen. $\Upsilon$ está demostradísimo que si tú tienes un ratón macho de laboratorio y lo castras antes de la pubertad, antes de que empiece a producir testosterona, va a tener un comportamiento reproductivo de hembra. Si este mismo ratón no lo castro durante la pubertad, pero lo castro después y le inyecto testosterona de una sola vez, su comportamiento reproductivo es de macho, por qué, porque durante la pubertad se moldean ciertos circuitos con estas hormonas que conllevan a ciertas respuestas. Esto lo digo asi porque a veces nos encontramos con opiniones en contra, que dicen que no, que todos somos iguales, y nosotros no compartimos eso, nosotros si creemos que somos distintos, y que somos complementarios, ahora, esto no quiere decir que vayamos a discriminar a alguien por su orientación sexual, bajo ningún punto de vista y no sólo no tenemos ningún problema con una persona que diga que tiene una orientación homosexual, así como tampoco tenemos ningún problema con una persona que diga que su orientación sexual es heterosexual, o sea, para nosotros son todos personas, $y$ son todos iguales en dignidad, sean hombre o mujer y sin importar tu orientación sexual. (Profesional, programa de educación sexual)

No hemos tenido un caso de algún alumno que se haya declarado abiertamente homosexual siendo alumno regular del colegio, sí tenemos ex alumnos que, más menos tres años después [de salir del colegio] han declarado su homosexualidad. Me da la impresión que estadísticamente es menor que en [el resto de] la población, yo creo que la homosexualidad debe ser más o menos el 1,0\%, el 1,5\% de la población, y nosotros debemos tener un 0,5\%, uno por cada dos generaciones más o menos al menos en los últimos diez años. La actitud frente a esos alumnos también... a ver, tampoco ellos han venido a decir, uno tampoco los va a ir a buscar no, son alumnos que han tenido siempre una postura más alejada del colegio, quizás más en ruptura con los valores que el colegio propone, entonces son alumnos que de por sí siempre han estado alejados o que una vez salidos del colegio rompieron el lazo. (Profesor, colegio nuevos movimientos católicos)

Aun cuando no se abandona la noción de "cuidado" de algún modo también se insinúa que la homosexualidad responde a una anomalía hormonal o valórica. Desde este punto de vista, es evidente que un individuo homosexual queda posicionado en un lugar de la diferencia, confirmándose con ello la razón por la cual no puede ser tratada con igualdad respecto a otras formas de sexualidad. Este tipo de explicaciones es criticada por los establecimientos más "progresistas" (definidos así por su oposición declarada con el pensamiento conservador) Aún si se abandona todo intento de explicación de la homosexualidad, para así comprender la "realidad del sujeto homosexual", al final se plantea una alternativa que podríamos denominar "inestable" pues no logra validar del todo la experiencia del individuo homosexual.

Las niñitas han ido evolucionando mucho más rápido que todos nosotros en todos esos temas, para ellas la homosexualidad casi que no es tema, y no entienden que la Iglesia no acepte que se casen los gays, o sea en eso las nuevas generaciones vienen obviamente mucho más abiertas $\boldsymbol{c}^{\boldsymbol{Y}}$ por qué crees tú que están más receptivas? Porque yo creo que se ha naturalizado, no es naturalizado la palabra, pero se ha ido como haciendo mucho más común, más corriente, se ha ido normalizando mucho más esta situación, aparece algo como más normal, los medios de comunicación yo creo que son muy fuertes, yo creo que hay una conciencia cada vez más clara de la dignidad de las personas y de los derechos. (...) Además, ellas 
ven casos en sus familias, de repente un primo... todas como que conocen a alguien [gay] que ubican y también ocurre que la gente ha perdido el temor a hablar también y como que se han ido sacando estos temas y no es una enfermedad. To creo que es mucho mejor que en otros tiempos en que ser maricón eran las penas del infierno, nadie se atrevía a abrir la boca porque te crucificaban po. (Profesora, colegio católico tradicional)

A pesar de que nuestra congregación es bien libertaria, acepta la posibilidad de que cada uno de los religiosos establezca lo que quiera frente al tema que quiera, pero frente a los temas trascendentales: aborto, embarazo no deseado, homosexualidad, diversidad de género, hay una línea editorial, o sea me tengo que regir por esto; y el alumno lo percibe, o sea, no... entonces el discurso de los religiosos de este colegio es un discurso fundamentalmente centrado en el amor, el amor como sentimiento de la afectividad máxima y con todas las consecuencias que pueda tener, es lo básico y principal y primordial, y su discurso del amor lo tienen ya institucionalizado, o sea "amor se describe como..." y "frente a una necesidad de amor puedes reaccionar de esta manera"; entonces el alumno lo ve distante, anacrónico. No, hoy día la diversidad es complejísima. (Profesor, colegio católico tradicional)

Este último extracto en particular nos expone a una cuestión ineludible en nuestro análisis: este discurso "aceptador" de la escuela necesariamente convive con el "rechazo" de la Iglesia hacia la homosexualidad expresado con claridad en el catecismo (Cfr. numerales 2357 y 2358). En este caso, es importante usar las comillas sobre ambas palabras pues esta tensión no puede analizarse situando magisterio y colegio, rechazo y aceptación como términos opuestos y absolutos. De acuerdo con el moralista Marciano Vidal (2010) "tanto la teología católica como la de otras confesiones cristianas han reflexionado sobre la relación entre cristianismo y homosexualidad a partir de las bases bíblicas y las tradiciones que cada magisterio impone en cuestión” (p. 143). Es probablemente la reproducción de este tipo de discusiones lo que permite a los establecimientos tratar la homosexualidad como "un tema para generar debate" y que permite a las estudiantes, por ejemplo, preguntarse sobre por qué "los gays no se pueden casar" o a los religiosos, el "centrarse en el amor".

Este tratamiento inestable y casuístico perpetúa el privilegio escondido que sigue teniendo la heterosexualidad. Aurélie Le Mat (2014) advierte además que la experiencia homosexual también es silenciada cuando la escuela afirma que aquella es finalmente un asunto privado cuando circunscribe la experiencia homosexual sólo a la orientación sexual (refiriéndose a las conductas íntimas) y no a la identidad sexual (socialmente reconocida, la cual es finalmente el "objeto" del debate). Esta afirmación de la autora nos hace interrogarnos respecto a cómo la postura progresista de algunos colegios puede asegurar la igualdad de condiciones el debate político con relación al rechazo magisterial de la homosexualidad, si al final la experiencia homosexual nunca es movilizada de la misma manera que la experiencia heterosexual, comparativamente mucho más pública y no cuestionada.

Independientemente de si el discurso es conservador o no, lo anterior impide desarrollar una posición reflexiva global respecto a cómo está siendo construida la sexualidad homosexual en el currículo. Decimos esto, porque el debate que hemos presentado en las citas nunca se relaciona con una reflexión sobre las condiciones efectivas que permiten o impiden la salida del closet de un estudiante al interior de la escuela y porque la homosexualidad es presentada de manera completamente desvinculada a la heterosexualidad, aun cuando a nivel general ambas categorías son producidas mutuamente todo el tiempo (Bozon, 2008). 
Debemos añadir un último elemento a propósito de esta presentación pública de la homosexualidad en la escuela. En un contexto donde el proyecto educativo prioriza la transmisión de valores, la elaboración de "modelos de persona", a través de una lógica testimonial donde el profesor de algún modo debe encarnar un ejemplo (Lacroix, 2010), todo aquello expresado por un educador no solo tiene un efecto discursivo sino que es asociado también a "quién es él/ella como persona". Para tener autoridad se debería ser creíble. De allí que no sea siempre fácil expresar la opinión personal, sobre todo cuando se está en contra del discurso magisterial que debe ser transmitido.

Pensamos que una de las maneras de solucionar este inconveniente es adoptar un tratamiento paternalista de la diferencia. Establecer una distancia con el "sujeto de cuidado" hace posible hablar de la homosexualidad sin que aquella comprometa del todo al hablante. Así se puede salvar bien esta posición inestable de los colegios "progresistas" que hemos presentado hasta ahora y también evitar el problema de discriminación abierta potencial que conlleva la aproximación conservadora.

\subsection{El paternalismo que silencia la alteridad}

Para evitar reducir lo que hemos expuesto a un comportamiento "hipócrita" de los actores, deberíamos siempre incluir la experiencia del hablante que se presenta a sí mismo como comprensivo dentro del marco de posibilidades concretas que brinda su entorno. Nos inclinamos más bien aquí a interpretar la ambigüedad que hemos presenciado a partir del paternalismo como posibilidad de adoptar una posición personal no comprometedora.

Entendemos el paternalismo a partir de la definición propuesta por Magni-Berton (2011) quien sostiene que el mismo es una forma de ejercicio político donde quien ocupa el poder asume para sí la responsabilidad que los individuos tengan una "buena vida", al mismo tiempo que sospecha sistemáticamente de la capacidad que aquellos cuentan con la capacidad suficiente de tomar buenas decisiones. Siguiendo al autor, las prescripciones propias del catecismo obedecen la mayor parte de las veces a lo que él mismo denomina "paternalismo puro" (donde la autoridad se ocupa de los objetivos que establecen para sí las personas y no de los medios para lograrlos).

Sin embargo, para no atribuir este rasgo únicamente a una cuestión de adhesión religiosa, podríamos incorporar el análisis del paternalismo escolar que realizan Dubet y Martuccelli (1996) en Sociologie de l'expérience scolaire. Los autores, a propósito de la función socializadora de la escuela, sostienen que aquella se define permanentemente por por la sumisión a la separación de saberes y la multiplicación de ejercicios, premios y exámenes, que tienen lugar dentro de una relación definida por la asimetría entre profesor y estudiante. Este carácter jerárquico unido al funcionamiento disciplinario de la institución, la convierten en un lugar propicio para un no reconocimiento de la capacidad de agencia del otro. En esto coinciden Silvie Ayral (2011) y Zoé Rollin (2012) quienes señala claramente como los espacios de care son momentos privilegiados para la expresión de discursos sexualizados en razón de la personalización de la labor docente: para ambas autoras, la administración de autoridad se estructura siempre en razón de una virilización del comportamiento que pocas veces se cuestiona y que al final impide ofrecer modelos de persona que escapen a lo que Pierre Bourdieu (2002) identificó como los principios de la dominación masculina (sumisión de lo no masculino a través del ejercicio de una violencia naturalizada). 
Es importante añadir aquí que la insistencia de la escuela católica de formar a través de modelos de virtud, de testigos, puede incurrir en los mismos inconvenientes paternalistas que suelen atravesar las relaciones de care. Tronto (2008) describe bien que la identificación de una necesidad -en este caso atender a la formación afectiva y sexual de los estudiantes enfrentados a la incertidumbre que genera la sexualidad- no siempre se condice con el modo como los sujetos conciben que dichas necesidades deben ser atendidas. El modelo de virtud no necesariamente está siempre al tanto de cómo es recibido. La relación desigual entre adultos y adolescentes o el resabio patriarcal que sospecha de las capacidades de los individuos de tomar sus propias decisiones, dan como resultado es un trato jerarquizado de los estudiantes pues siempre se puede asumir que ellos no son completamente propietarios de sí (Leite, 2013)

Por ejemplo un alumno en tercero medio se declaró gay y eso generó en los jóvenes
una respuesta en dos grupos. Los que se incomodaron con la declaración pública de
este niño y los que inmediatamente entraron en la validación del acto que él había
tenido. Porque hoy en día los jóvenes están cien pasos más adelante que los adultos
en términos de valorar la diversidad, con dos subgrupos, el que valora la diversidad
desde la indiferencia, "sabéis que me da lo mismo lo que haga este, me da lo mismo
lo que haga cualquiera, que cada uno haga lo suyo”, que para mí más que un valor
es una posición súper cómoda, muy light, muy posmoderna, hasta la que es realmente
tolerante, que inclusive asume sus propias interrogantes como 'ichuta, qué me voy a
cambiar en Educación Física al lado de él!, voy a ir al viaje de estudios con él, va a
dormir en mi pieza! (Orientador, colegio nuevos movimientos católicos)

Aquí el paternalismo se hace evidente en este modo de juzgar las reacciones que tienen los estudiantes, clasificándolas a partir de un esquema propio que de algún modo insinúa aquellas respuestas deseables de aquellas que no (no es neutro ser "posmoderno" en una escuela católica) Este paternalismo sumado a la necesidad de abordar la sexualidad desde una perspectiva de cuidado termina por anular la capacidad de agencia del sujeto homosexual que tomó la decisión de salir del clóset (decisión que por lo demás nunca está construida sólo por disposiciones personales); al mismo tiempo, evita que el hablante se cuestione respecto a la estructura moral compleja con la que está posicionando la reacción de los otros individuos “postmodernos” y “tolerantes".

La cultura y todos nosotros hemos de alguna manera hemos normalizado la homosexualidad como una conducta o una orientación sexual válida dentro de la sociedad, absolutamente igualada! pero en el inconsciente o en el relato cultural de la masculinidad la homosexualidad sigue siendo una sombra. ¿ $\Upsilon$ qué es lo que pasa? que el cabro que no tiene relaciones sexuales con la polola se le comienza a generar a él mismo y a los amigos la duda, ¿̇y cuál es la duda? La duda no es que tú seas reprimido, no es que tú estés esperando, perdón, la duda es que seas homosexual! ¿Por qué eso te parece tan políticamente incorrecto de decir? Creo que esta sociedad de los fragmentos en el fondo [es una sociedad que] presenta una especie de realidad construida en fragmentos de verdades, que genera una cosa que es súper importante, que es como este paraguas donde cabe todo lo que de alguna manera podría ser llamado tolerancia, pero esa tolerancia es mucho más aparente que real. Jugamos a que no somos homofóbicos, jugamos a que el padre y la madre soltera [están bien]... jugamos que no nos importa tu pasado, de dónde viniste, dónde estudiaste. Mentira! porque para los créditos de los bancos te preguntan dónde vives y en qué colegio estudiaste. Entonces jugamos con una serie de cosas, pero que son cosas que no se pueden decir (...) Mira el gran aumento de conductas lésbicas en las mujeres, fundamentalmente tiene a la base un miedo a los hombres, ahi no hay un tema de que 'ella es lesbiana'. Mentira! a ella lo que le pasa tiene una herida que le da terror enfrentarse a los hombres, entonces si ella tiene quince años no le pongamos ninguna etiqueta, veamos qué hay detrás de esa conducta. Lo mismo que otra niñita de quince años que es súper heterosexual, pero que cambia pololos todas las semanas, 
o sea, si no es malo pololear, pero veamos ¿̇estai bien, estai tranquila? Eso está indicando algo, sobre todo cuando la conducta es exagerada o minimizada (sic), siempre los extremos te están indicando algo. Con este cuento de la homofobia y de la tolerancia, no nos estamos dando cuenta que son síntomas, es lo mismo que le han pasado a los psicólogos con respecto a la masturbación, de normalizarla a tal punto de no darse cuenta que muchas veces cuando se transforma en una conducta habitual está hablando algo mucho más profundo como soledad angustia, ansiedad, una manera de compensar. (Profesional, programa de educación sexual)

A nuestro juicio, esta larga cita sintetiza nuevamente las consecuencias de la aproximación paternalista hacia la homosexualidad en el espacio escolar: aquí se anula la capacidad de agencia del individuo que es etiquetado como "distinto", independientemente de la identidad que se le asigne: el gay, la lesbiana, la muchacha que cambia de pololos, el adolescente que se masturba. Para quien habla la conducta visible es en realidad el resultado de un problema "invisible", todo lo cual por añadidura le hace sospechar de la retórica de la tolerancia que estaría instalada en el espacio escolar actual. Lo que resulta complejo de esta sospecha es que no se pregunta sobre aquellas condiciones que afectan el modo como un sujeto toma decisiones; en vez de ello prefiere corregir una identidad "problemática". Este tipo de aproximaciones finalmente no permite desarrollar una reflexión exhaustiva sobre el sistema general de construcción de la sexualidad contemporánea, perpetuando la jerarquía de identidades dentro de la escuela.

Si bien la cita corresponde a una organización conservadora, es importante señalar que aquella presta servicio a varios colegios que formaron parte de nuestra muestra, incluyendo algunos que se definieron como progresistas. Esto significa que en cierta medida hay un principio que hace converger los discursos, independientemente de cómo se aborda la "cuestión homosexual". Reiteramos que el paternalismo sería dicho punto de encuentro. Quizás por esta misma razón, la condescendencia mostrada en la cita anterior de algún modo se replica entre los estudiantes de un colegio católico tradicional, quienes a través de una operación de diferenciación de algún modo reconocen al otro como un alter, pero le restan toda capacidad de interpelar la norma principal en la que se desenvuelven.

\begin{abstract}
Siempre he dicho que quiero un amigo gay para preguntarle un montón de cosas que desconozco y lo admito. Algunos dicen que se nace gay y otros no se nace y la única manera de saber es preguntándole a una persona homosexual asumida, tampoco que lo exponga, pero que se encarguen de investigar, de preguntar a alguien y eso entregarlo a nosotros. A mí me interesa mucho el tema de la homosexualidad siendo que yo soy hetero, pero me interesa mucho porque son personas que por amar a alguien del mismo sexo son súper rechazadas. (...) yo en mi ignorancia yo o sea, en mi ignorancia y en el estereotipo que te sumergen, ya si es medio afeminado es gay, no, no es así, y eso encuentro que también debieran enseñarlo. También deberían entregar información, porque tú no puedes llegar y preguntar a una persona hetero ¿los gay nacen o se hacen? Los profesores también ahí desconocen información desde el lado cristiano les cuesta asumirlos también, no vengan con cosas, a la Iglesia le cuesta admitir dejar que se casen, por la iglesia digo. También hay un tema de los homosexuales con la Iglesia que también sería interesante verlo desde el lado de la Iglesia y desde no el lado de la Iglesia. No por ser cristiano la única verdad es la iglesia, sino que también nos den las opciones de saber, de ver, de conocer. (Estudiante, grupo focal colegio católico tradicional)
\end{abstract}

La buena disposición de los jóvenes se confronta necesariamente con este límite difuso que posiciona la homosexualidad en un plano de no igualdad, aún si como señala la estudiante "el cristiano debería abrirse a la comprensión de ese otro". La norma eclesial 
personalista es reinterpretada en clave responsabilidad individual ("es necesario saber más") pero también interpersonal ("aceptar, conmoverse con el que ha sido rechazado"). Sin embargo, saber lo que le pasa a ese otro no significa necesariamente reflexionar respecto a cómo dicha experiencia necesariamente repercute en el propio proceso de individuación sexual. La estudiante nunca se pregunta sobre cómo se reconoce la atracción por el mismo sexo o sobre cómo el estereotipo puede determinar estrategias de agenciamiento de la sexualidad puesta del lado de la diferencia. Si todos los individuos son interpelados por la misma norma de coherencia sexual, este tipo de preguntas serían fundamentales a la hora de desarrollar una educación sexual reflexiva así como también para evitar la discriminación. Y para responderlas se precisa que el otro sea reconocido en su totalidad como individuo.

\section{Conclusión}

En relación a la normalidad, Phil Hubbard (2002) reflexiona en torno a los mecanismos sociales que permiten la reproducción de lo que él denomina una "buena ciudadanía" heterosexual. Esto, porque siempre hay formas de diferenciación entre las conductas sexuales permitidas y no permitidas en cualquier espacio público, modos de recluir el deseo "peligroso" o desconocido, maneras de invisibilizar al sujeto "desviado", canales para sospechar de la coherencia ajena en materia de sexualidad. En dicho contexto, aparece indefectiblemente la pregunta sobre quién tendría y cómo se expresaría la mejor coherencia sexual que se requiere para ser ese "buen ciudadano": quién encarna las normas, quién las evalúa, quién es capaz de describirlas de manera reflexiva.

Por la misma razón, no podemos finalizar nuestro análisis sin señalar que todavía falta averiguar como la capacidad de agencia se reivindica por parte de los mismos sujetos a veces minimizados. Dadas las características de nuestro trabajo de campo esto no ha sido posible (precisamente porque la comprensión de la homosexualidad ligada a eventos o a un relativo silenciamiento de los individuos hace muy complejo acceder a opiniones de primera fuente). No obstante podemos adelantar que, independientemente del sujeto que entrevistáramos, la capacidad de agencia y el componente reflexivo de la propia sexualidad se expresarían en la percepción de una distancia entre cómo se transmiten las normas y como aquello interpela la "realidad que a uno le toca vivir" (Dubet, 2010). De algún modo, en esta crítica de su propia posición, el sujeto se presenta como un individuo irreductible y consciente de sí mismo a la vez.

Teniendo esto en consideración, resulta problemático que la lógica de cuidado y aceptación de la homosexualidad en las escuelas observadas no se interrogue sobre la capacidad de agencia de dichos individuos o sobre las condiciones que permitirían ejercerla públicamente. En otras palabras, el debate sobre el rechazo eclesial o sobre cualquier otro problema que afecte a los individuos homosexuales es incompleto, a menos que se incorpore la pregunta sobre cómo dicha persona ha abordado su sexualidad asegurando su coherencia e incorporando positiva o negativamente tanto las experiencias como las miradas de los individuos heterosexuales (predominantes en el espacio que examinamos). Al mismo tiempo, la educación sexual y afectiva "reflexiva" es incompleta si no vislumbra que la heterosexualidad dominante está permanentemente siendo producida por la delimitación que la escuela hace de la homosexualidad. 
Lo anterior es fundamental pues postulamos que cualquier trabajo del individuo sobre sí mismo no es un trabajo que esté fuera de un proceso de socialización. De allí que no se comprenda la responsabilidad y la coherencia individual si no se analiza también cómo las distinciones entre lo normal y lo diferente influyen sobre la subjetividad personal. Tal como señala Francois de Singly (2010) cuando se quiere comprender lo que ocurre en el ámbito de la experiencia se debe atender al juego de miradas que existe entre un Ego y un Alter, soberanos y autónomos aunque no necesariamente simétricos, todos los cuales observan aquí una coherencia afectiva propia y del otro. Tomar conciencia de esta relación, implica reivindicar necesariamente la capacidad de agencia individual de cada término. Ciertamente esto es algo que el discurso magisterial difícilmente permitirá, pero la escuela católica no se limita en caso alguno a reproducir únicamente dicho discurso.

Como lugar de reproducción del conocimiento, como institución que apela a la formación de los individuos insertos en su cultura, se debería garantizar al menos que todos los individuos enfrenten de igual manera la exigencia de coherencia sexual que caracteriza la sexualidad moderna. La crítica respecto a cómo persisten las desigualdades para llevar a cabo dicho proceso, más que la negación o la aceptación incompleta de quien no parece cumplir las exigencias, bien puede ser la forma como las escuelas católicas dan cumplimiento a uno de sus propósitos institucionales: enseñar la aceptación incondicional del otro.

\section{Referencias}

Apablaza, M. (2015). El orden en la producción de conocimiento: normatividades en la educación chilena en torno a la diversidad. Revista de Estudios Pedagógicos, 41, 253-266. doi:10.4067/s0718-07052015000300016

Ayral, S. (2011). La fabrique des garçons. Sanctions et genre au collège. París: Presses universitaires de France.

Barrientos, J. (2015). Violencia homofóbica en América Latina y Chile. Santiago: El Desconcierto.

Borrillo, D. (2001). Homofobia. Barcelona: Bellaterra

Bourdieu, P. (2002). La domination masculine. París: Editions du Seuil.

Bozon, M. (2001a). Les cadres sociaux de la sexualité. Sociétés Contemporaines, 41, 5-9. doi: $10.3917 /$ soco.041.0005

Bozon, M. (2001b). Orientations intimes et construction de soi. Pluralité et divergences dans les expressions de la sexualité. Sociétés Contemporaines, 41(1), 11-40. doi: $10.3917 /$ soco.041.0011

Bozon, M. (2008). Les minorités sexuelles, sont-elles l'avenir de l'humanité? En V. Descoutres, M. Digoix, E. Fassin y W. Rault, (Coords.), Mariages et homosexualités dans le monde. L'arrangement des normes familiales (pp. 190-202). París: Autrement.

Bozon, M. (2009). Sociologie de la sexualité. París: Armand Colin.

Cáceres, C. y Salazar, X. (2013). Era como ir todos los días al matadero... El bullying homofóbico en instituciones públicas de Chile, Guatemala y Perú. Lima: UNESCO.

Canales, M. (2013). Análisis sociológico del habla. En M. Canales (Coord.), Escucha de la escucha. Análisis e interpretación en la investigación cualitativa (pp. 171-188). Santiago: Lom. 
Ceballos, M. (2012). Indicadores aplicados a la visión dominante de la masculinidad por adolescentes de educación secundaria: la importancia del "deber ser hombre". Última Década, 36(20), 141-162. doi:10.4067/s0718-22362012000100007

De Miguel, A. (2015). Neoliberalismo sexual. El mito de la libre elección. Madrid: Cátedra.

De Singly, F. (2000). Libres ensemble. L'individualisme dans la vie commune. París: Nathan

De Singly, F. (2010). Les uns avec les autres. Quand l'individualisme crée du lien. París: Pluriel.

Devis, J., Fuentes, J. y Sparkes, A. (2005). ¿Qué permanece oculto en el currículum oculto? Las identidades de género y de sexualidad en la educación física. Revista Iberoamericana de Educación, 39, 73-90.

Dubet, F. (2010). La sociología de la experiencia. Madrid: Editorial Complutense.

Dubet, F. y Martuccelli, D. (1996). A l'école. Sociologie de l'expérience scolaire. París: Seuil.

Fassin, E. (2008). L’inversion de la question homosexuelle. París: Amsterdam Press.

Generelo, J. y Pichardo, J. (2006). Homofobia en el sistema educativo. Madrid: COGAM.

Hubbard, P. (2002). Sexing the self: geographies of engagement and encounter. Social and Cultural Geography, 3(4), 365-381. doi:10.1080/146493602 1000032478

Illouz, E (2015). Why love hurts. A sociological explanation. Cambridge: Polity Press.

Kaufmann, J. C. (2011). Sociologie du couple. París: Presses universitaires de France.

Lacroix, X. (2010). Autorité et affectivité dans la famille. En D. Avanzini (Coord.), Affectivité et autorité en éducation (pp. 43-64). París: Don Bosco.

Le Mat, A. (2014). L’homosexualité, une «question difficile». Distinction et hiérarchisation des sexualités dans l'éducation sexuelle en milieu scolaire. Genre, Sexualité et Société, 11, 31-44. doi: $10.4000 /$ gss.3144

Leite, V. (2013). Sexualidade adolescente como direito? A visão de formuladores de políticas públicas. Rio de Janeiro: Universidade do Estado do Rio de Janeiro.

Madrid, S. (2013). The formation of ruling class men: private schooling, class and gender relations in contemporary Chile (Tesis doctoral). University of Sydney, Australia.

Magni-Berton, R. (2011). Care, paternalisme et vertu dans une perspective libérale. Raisons Politiques, 44(4), 139-162. doi:10.3917/rai.044.0139

Martino, W. (1999). 'Cool boys', 'party animals', 'squids' and 'poofters'. Interrogating the dynamics and politics of adolescent masculinities in school. British Journal of Sociology of Education, 20(2), 239, 263. doi:10.1080/01425699995434

Matus, C. e Infante, M. (2011). Undoing diversity: knowledge and neoliberal discourses in colleges of education. Discourse-Studies in the Cultural Politics of Education, 32(3), 293-307. doi:10.1080/01596306.2011.573248

Palma, I., Reyes, D. y Moreno, C. (2013). Educación sexual en Chile. Pluralismo y libertad de elección que esconde una propuesta gubernamental conservadora. Política educativa, 49, $14-24$.

Renold, E. (2004). 'Other' boys: negotiating non-hegemonic masculinities in the primary school. Gender and Education, 16(2), 247-266. doi:10.1080/09540250310001690609

Rollin, Z. (2012). Genre et sexualité dans le rapport pédagogique : ethnographie d'un lycée «de banlieue». Genre, Sexualité et Société, 7, 23-50. doi:10.4000/gss.2350 
Scott, J. (2014). Emancipation et égalité, une généalogie critique. Recuperado de http://www.contretemps.eu/

Sevegrand, M. (2002). Jean-Paul II et la sexualité. En J. Maître y G. Michelat (Eds.), Religion et sexualité (pp. 217-228). Paris: L'Harmattan.

Thumala, M. A. (2007). Riqueza y piedad. El catolicismo de la élite económica chilena. Santiago: Debate.

Tronto, J. (2008). Du care. Revue du MAUSS, 32(2), 243-265. doi:10.3917/rdm.032.0243

Vidal, M. (2010). Sexualidad y condición homosexual en la moral cristiana. Buenos Aires: San Pablo

\section{Breve CV del autor}

\section{Pablo Astudillo Lizama}

Sociólogo, Máster en sociología, especialidad Género, Política y Sexualidad de la Escuela de Altos Estudios en Ciencias Sociales (EHESS) de París. Doctorante en sociología de la Universidad Paris V. Tesista asociado al Centre de Recherche sur les Liens Sociaux (CERLIS), especializado en temáticas de género, sexualidad, diferenciación social y educación y al proyecto Anillo de Investigación Conicyt "Normalidad, Diferencia, Educación". Ha publicado artículos en relación a la diferenciación social en los espacios de sociabilidad homosexual y actualmente se ha especializado en cuestiones relativas a la educación sexual y afectiva en escuelas católicas de élite en Santiago de Chile. Email: pablo.astudillo.lizama@gmail.com 